\title{
A New Four-Scroll Chaotic Attractor Consisted of Two-Scroll Transient Chaotic and Two-Scroll Ultimate Chaotic
}

\author{
Yuhua $X u_{,}^{1,2,3}$ Bing Li, $^{4}$ Yuling Wang, ${ }^{5}$ \\ Wuneng Zhou, ${ }^{3}$ and Jian-an Fang ${ }^{3}$ \\ ${ }^{1}$ Department of Mathematics and Finance, Yunyang Teachers' College, Hubei Shiyan 442000, China \\ ${ }^{2}$ Computer School of Wuhan University, Wuhan 430079, China \\ ${ }^{3}$ College of Information Science and Technology, Donghua University, Shanghai 201620, China \\ ${ }^{4}$ NOSTA, The Ministry of Science and Technology of China, GPO Box 2143, Beijing 100045, \\ Tianjin University, Tianjin 300072, China \\ ${ }^{5}$ School of Management, Tianjin University, Tianjin 300072, China
}

Correspondence should be addressed to Yuhua Xu, yuhuaxu2004@163.com

Received 21 January 2012; Accepted 12 March 2012

Academic Editor: Ahmad M. Harb

Copyright (C) 2012 Yuhua Xu et al. This is an open access article distributed under the Creative Commons Attribution License, which permits unrestricted use, distribution, and reproduction in any medium, provided the original work is properly cited.

\begin{abstract}
A new four-scroll chaotic attractor is found by feedback controlling method in this paper. The novel chaotic system can generate four scrolls two of which are transient chaotic and the other two of which are ultimate chaotic. Of particular interest is that this novel chaotic system can generate one-scroll, two 2-scroll and four-scroll chaotic attractor with variation of a single parameter. We analyze the new system by means of phase portraits, Lyapunov exponents, fractional dimension, bifurcation diagram, and Poincaré map, respectively. The analysis results show clearly that this is a new chaotic system which deserves further detailed investigation.
\end{abstract}

\section{Introduction}

Since Lorenz found the first chaotic attractor, considerable research interests have been made in searching for new chaotic attractors. Particularly, research interests are turning in searching for new chaotic attractors in the three-dimensional (3D) autonomous ordinary differential equations. For example, Lorenz system [1], Rössler system [2], Chen and Ueta system [3], Lü and Chen system [4], and Liu system [5] were reported and analyzed.

In very recent years, creating complex multiscroll or multiwing chaotic attractors in $3 \mathrm{D}$ autonomous systems has been rapidly developed [6, 7]. It stimulates the current research interest in creating various complex multiscroll chaotic attractors by using simple electronic circuits and devices. After the rapid development in more than a decade, multiscroll chaotic 
attractors generation has become a relatively mature research direction [8]. In fact, most of the multiscroll attractors were generated by increasing the breakpoints in the nonlinearity. Recently, a four-wing or three-wing attractor was generated in some 3D systems by relying on two embedded state-controlled binary switches [9]. But these 3D systems are not usually smooth systems. Although a few 3D smooth autonomous chaotic systems have been reported to display two-, three-, and four-wing attractor, respectively [10-17], how to generate multiwing chaotic attractors remains an open problem. Therefore, it is important and even necessary to investigate various possible chaotic behaviors such that we can establish a unified theory for a 3D system generating chaos.

Particularly, over the last two decades chaos in engineering systems, such as nonlinear circuits, has gradually been moved from simply being a scientific curiosity to a promising subject with practical significance and applications. Recently, it has been noticed that purposefully creating chaos can be a key issue in many technological applications such as communication, encryption, and information storage. An appropriate system for such applications could be chosen from a category of easier controlled chaotic system to optimize factors such as robustness to errors in the parameters or immunity to noise [18]. Therefore, it is obviously significant to create more complicated chaotic systems with simple expressions in three-dimensional for engineering applications such as secure communications.

In this paper, we propose a four-scroll chaotic attractor which consists of the twoscroll transient chaotic and the two-scroll chaotic. It is very desirable for engineering applications such as secure communications. For example, in terms of decryption, we can generate a certain degree of confusion by using chaos system of transient chaos feature to encrypt. Moreover, this novel system can generate one-scroll, left two-scroll, right twoscroll (according to the system's geometric locations), and four-scroll chaotic attractors, respectively, with the variation of a single parameter. This paper is devoted to a more detailed analysis of this new chaotic attractor.

\section{New Chaotic System}

Based on the chaotification analysis $[18,19]$, the Liu-Chen system may be added with two terms, which leads to the finding of the new chaotic attractor.

Start with the controlled Liu-Chen system [10]:

$$
\begin{gathered}
\dot{x}=b x+c y z+u_{1}, \\
\dot{y}=d y+e x z+u_{2}, \\
\dot{z}=f z+g x y .
\end{gathered}
$$

By various trial tests, we find a simpler anticontroller, that is,

$$
\begin{aligned}
& u_{1}=a y, \\
& u_{2}=-z,
\end{aligned}
$$

which yields the following new chaotic system:

$$
\begin{gathered}
\dot{x}=a y+b x+c y z, \\
\dot{y}=d y-z+e x z, \\
\dot{z}=f z+g x y,
\end{gathered}
$$

where $[x(t), y(t), z(t)]^{T} \in R^{3}$ is the state vector and $a, b, c, d, e, f$, and $g$ are real constants. 
This new system (2.3) is found to be chaotic in a wide parameter range and possesses many interesting complex dynamical behaviors. For example, the system (2.3) can generate four-scroll for the parameters $a=2.4, b=-3, c=14, d=-11, e=4, f=5.85, g=-1$ and the initial conditions $[1,3,5]$ (see Figure 1(a)).

Sprott [17] has suggested that the long calculation time helps ensure that the solutions are steady states. Notably, in comparison with those of existing two-scroll or four-scroll chaotic attractors in 3D autonomous systems, the novel chaotic attractor can generate fourscroll consisted of the two-scroll transient chaotic and the two-scroll chaotic (see Figures 1(b)$1(\mathrm{~d})$ ). After time $t>55$, simulation results show that the attractor is no longer a four-scroll attractor and becomes a two-scroll attractor.

\section{Some Basic Properties of the New System}

In this section, we will investigate some basic properties of the new system (2.3).

\subsection{The Four-Scroll Chaotic Attractor}

\subsubsection{Equilibria}

It is known that the number of equilibrium points of the system and the stabilities at the equilibrium points are very important for the emergence of chaos. In the sequel, we consider the equilibrium points of system (2.3).

Let

$$
\begin{gathered}
a y+b x+c y z=0, \\
d y-z+e x z=0, \\
f z+g x y=0 .
\end{gathered}
$$

Let $a=2.4, b=-3, c=14, d=-11, e=4, f=5.85, g=-1$. Equation (3.1) has five equilibrium points as follows:

$$
\begin{gathered}
E_{1}(0,0,0), \\
E_{2}(4.1379,1.0050,0.7109), \\
E_{3}(-3.8879,1.2560,-0.8347), \\
E_{4}(-3.8879-0.9981,0.6633), \\
E_{5}(4.1379,-1.2473,-0.8823) .
\end{gathered}
$$

As shown in Figure 2, the equilibria points of system (2.3), $E_{2}, E_{3}, E_{4}$, and $E_{5}$ are located at the center of the four wings of the attractor, respectively, and the origin $\left(E_{1}\right)$ is located at the center of whole chaotic attractor. Moreover, it can be seen from Figure 2 that the equilibria $E_{2}, E_{5}$ play an important role in generating the two-scroll transients chaotic, while the equilibria $E_{3}$ and $E_{4}$ play an important role in ultimate generating two-scroll chaotic attractor. 


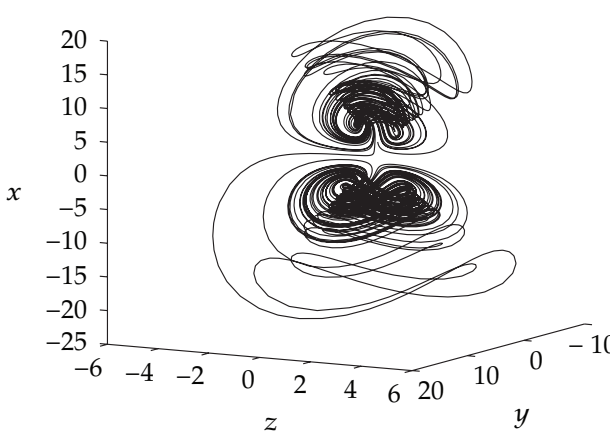

(a)

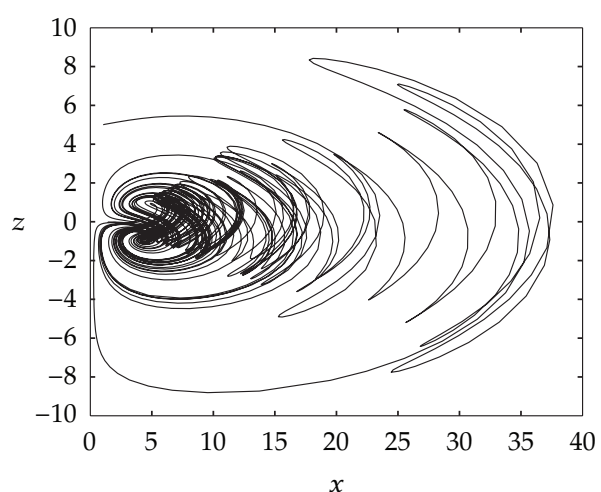

(c)

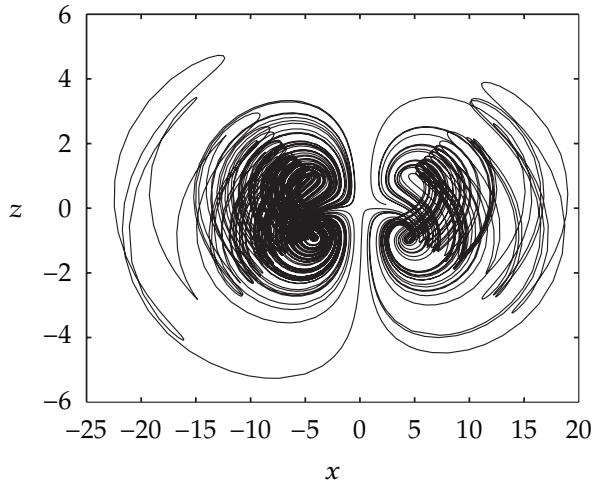

(b)

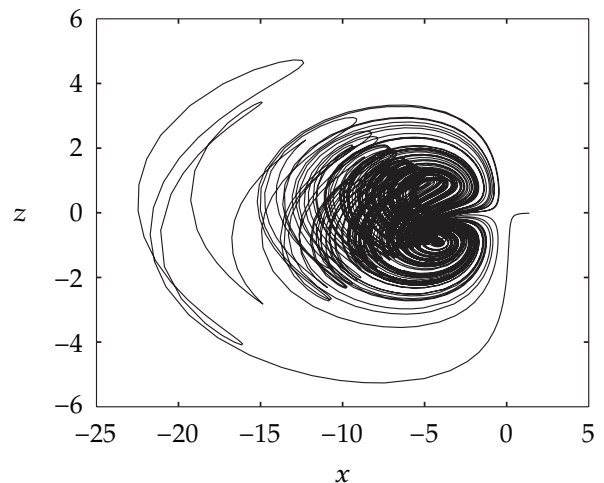

(d)

Figure 1: (a) The new chaotic attractor. (b) $x$ - $z$ phase plane of the new chaotic attractor. (c) When time $0<t \leq 55$, the new system generate two-scroll transient chaotic. (d) When time $t>55$, the new system generate two-scroll chaotic attractor.

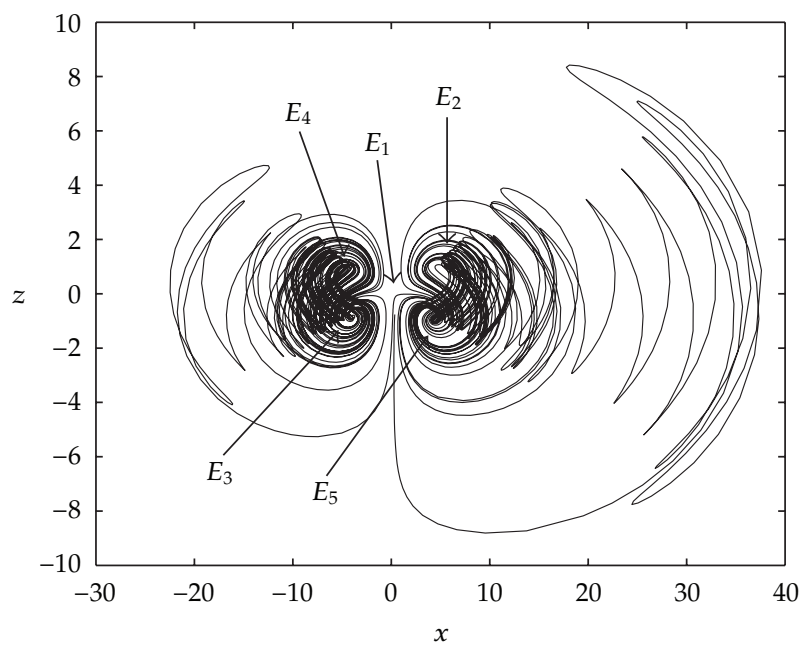

Figure 2: Five equilibria of the new four-scroll chaotic attractor. 

follows:

For equilibrium $E_{1}$, the system (2.3) is linearized, and the Jacobian matrix at $E_{1}$ is as

$$
J=\left(\begin{array}{ccc}
b & a+c z & c y \\
e z & d & e x-1 \\
g y & g x & f
\end{array}\right)=\left(\begin{array}{ccc}
-3 & 2.4 & 0 \\
0 & -11 & -1 \\
0 & 0 & 5.85
\end{array}\right)
$$

To gain its eigenvalues, we let $|\lambda I-J|=0$.

So the corresponding eigenvalues at $E_{1}$ are

$$
\lambda_{1}=-3, \quad \lambda_{2}=-11, \quad \lambda_{3}=5.85 .
$$

Similarly, the corresponding eigenvalues at $E_{2}$ are

$$
\lambda_{1}=-12.9021, \quad \lambda_{2}=2.3761+7.0804 i, \quad \lambda_{3}=2.3761-7.0804 i .
$$

The corresponding eigenvalues at $E_{3}$ are

$$
\lambda_{1}=-12.8005, \quad \lambda_{2}=2.3252+7.7881 i, \quad \lambda_{3}=2.3252-7.7881 i .
$$

The corresponding eigenvalues at $E_{4}$ are

$$
\lambda_{1}=-12.5469, \quad \lambda_{2}=2.1984+6.9804 i, \quad \lambda_{3}=2.1984-6.9804 i .
$$

The corresponding eigenvalues at $E_{5}$ are

$$
\lambda_{1}=-13.1525, \quad \lambda_{2}=2.5013+7.8516 i, \quad \lambda_{3}=2.5013-7.8516 i .
$$

From (3.4)-(3.8), we know that $E_{1}, E_{2}, E_{3}, E_{4}$, and $E_{5}$ are all unstable saddle points.

\subsubsection{Dissipativity and the Existence of Attractor}

For dynamical system (2.3), we can obtain

$$
\nabla \cdot V=\frac{\partial \dot{x}}{\partial x}+\frac{\partial \dot{y}}{\partial y}+\frac{\partial \dot{z}}{\partial z}=b+d+f
$$

where $b+d+f=-8.15$ is a negative value. Dynamical system (2.3) is a dissipative system, and an exponential contraction of the system (2.3) is

$$
\frac{d V}{d t}=e^{-8.15}
$$

In the dynamical system (2.3), a volume element $V_{0}$ is apparently contracted by the flow into a volume element $V_{0} e^{-8.15 t}$ in time $t$. It means that each volume containing the trajectory of this dynamical system shrinks to zero as $t \rightarrow \infty$ at an exponential rate -8.15 . So, all this dynamical system orbits are eventually confined to a specific subset that have zero volume, and the asymptotic motion settles onto an attractor of the system (2.3). 


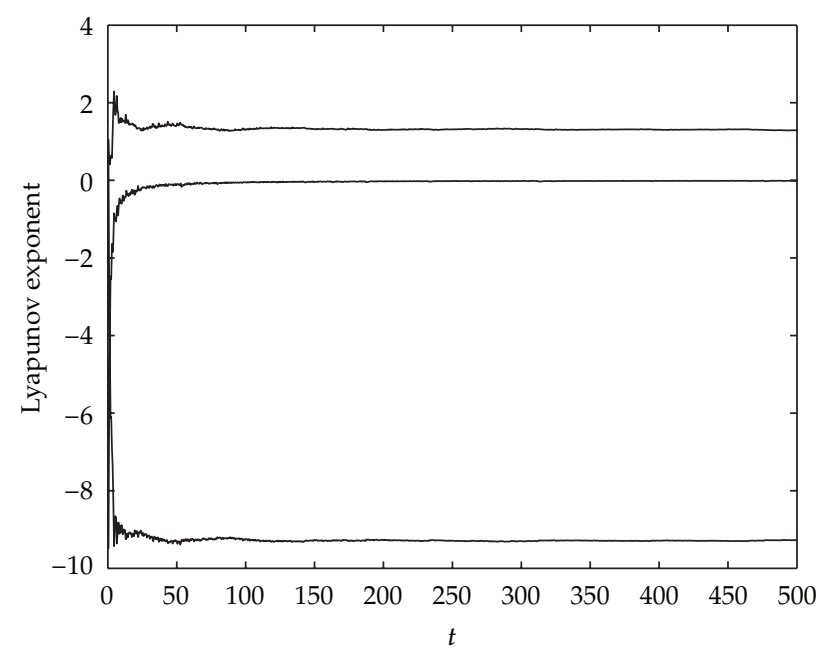

Figure 3: Lyapunov exponents.

\subsubsection{Lyapunov Exponents and Spectrum Map}

Any system containing at least one positive Lyapunov exponents is defined to be chaotic [20]. The Lyapunov exponent spectrum of the system (2.3) is found to be $L_{1}=2.8063, L_{2}=-0.0050$, $L_{3}=-2.8020$ (see Figure 3). In addition, the Lyapunov dimension of this system is

$$
\begin{aligned}
D_{L} & =j+\frac{1}{\left|L_{j+1}\right|} \sum_{i=1}^{j} L_{i}=2+\frac{L_{1}+L_{2}}{\left|L_{3}\right|} \\
& =2+\frac{2.8013}{|-2.8020|}=2.9998,
\end{aligned}
$$

which means that the system (2.3) is really a dissipative system, and the Lyapunov dimension of this system is fractional. The fractal nature of an attractor does not merely mean this system has nonperiodic orbits; it also causes nearby trajectories to diverge.

We can further find that the spectrum of system (2.3) exhibits a continuous broadband feature as shown in Figure 4.

\subsubsection{Forming Mechanism of the Four-Scroll Chaotic Attractor Structure}

In order to reveal the forming mechanism of the four-scroll chaotic attractor structure, a controlled system is proposed. The autonomous differential equations of this controlled system are expressed as

$$
\begin{aligned}
& \dot{x}=a y+b x+c y z, \\
& \dot{y}=d y-z+e x z, \\
& \dot{z}=f z+g x y+u .
\end{aligned}
$$

In this system, $u$ is a parameter of control and the value of $u$ can be changed within a certain range. 


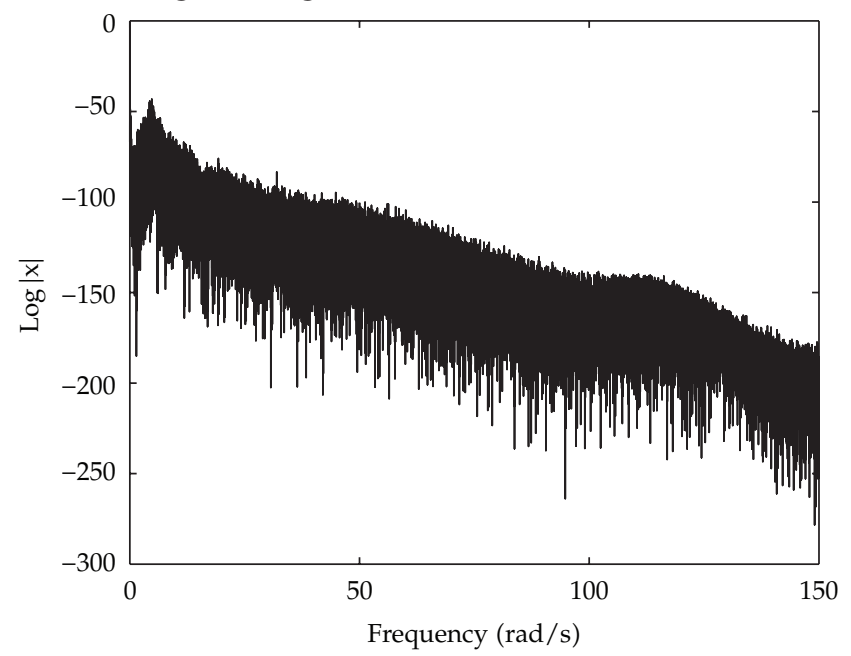

Figure 4: An apparently continuous broadband frequency spectrum Log $|x|$.

When the parameter $u$ is changed, the chaos behavior of this system can effectively be controlled. So it is a controller. In the numerical simulation, the initial values of the system $(3.12)$ is $(1,3,5)$.

For $u=-9$, the attractor evolves into the limit cycles; the limit cycles are shown in Figure 5(a).

For $u=-5.11$, the attractor evolves into the period-doubling bifurcations; perioddoubling bifurcations are shown in Figure 5(b).

For $u=-1.94$, the corresponding strange attractors are shown in Figure 5(c). Moreover the attractors are evolved into the one lower-scroll attractor.

For $u=-0.45$, the strange attractors are shown in Figure 5(d), the attractor evolves into the single left two-scroll attractor.

For $u=0.45$, the corresponding strange attractors are shown in Figure 5(e); the attractor evolves also into the single right two-scroll attractor.

For $u=1.94$, the corresponding strange attractors are shown in Figure 5(f). Moreover, the attractors are evolved into the one upper-scroll attractor.

For $u=5.11$, the attractor evolves into the period-doubling bifurcations; perioddoubling bifurcations are shown in Figure 5(g).

For $u=9$, the attractor evolves into the limit cycles; the limit cycles are shown in Figure 5(h).

In the controller, one can see that when $|u|$ is large enough, chaos attractor disappears; when $|u|$ is small enough, a complete chaos attractor appears. So $|u|$ is an important parameter to control chaos in the nonlinear system.

For $-9 \leq u \leq 9$ the bifurcation diagram of system (3.12) shows the complicated bifurcation phenomena (see Figure 6). It is clear that the bifurcation phenomenon well coincides with the forming mechanism.

\subsection{The One-Scroll Chaotic Attractor}

The system (2.3) has been found to generate a one-scroll chaotic attractor by only varying a single parameter. Here, the parameter $f$ is selected to be varied. For example, if we let $a=2.4$, $b=-3, c=14, d=-11, e=4, f=7, g=-1$ then a one-scroll chaotic attractor can be observed, as depicted in Figure 7. 


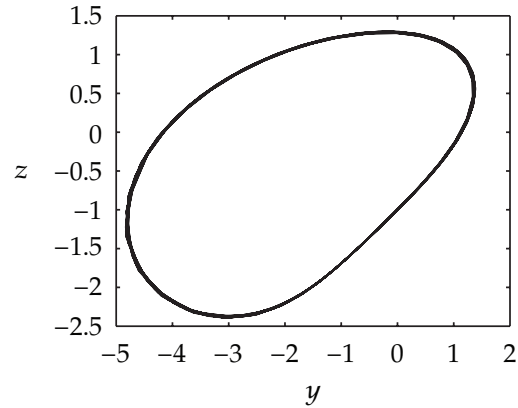

(a)

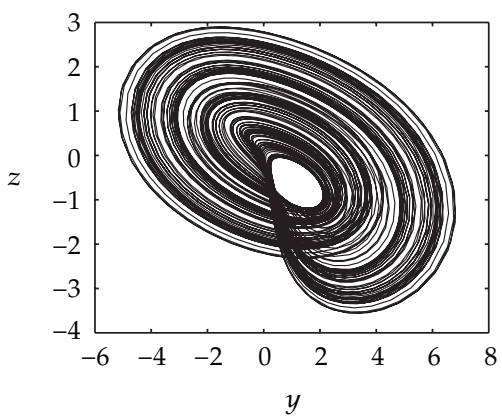

(c)

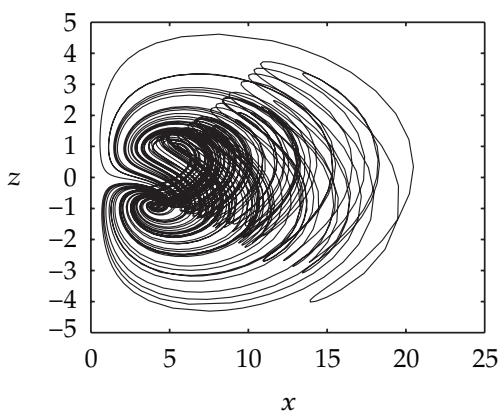

(e)

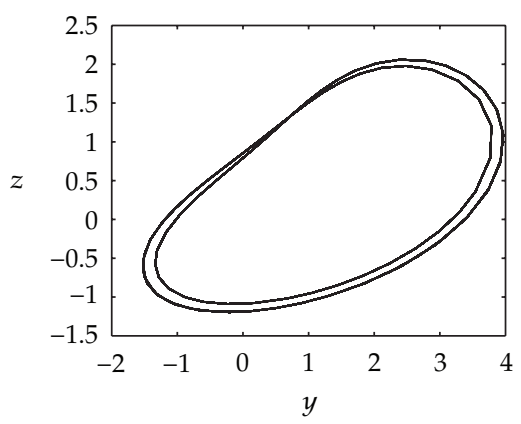

(g)

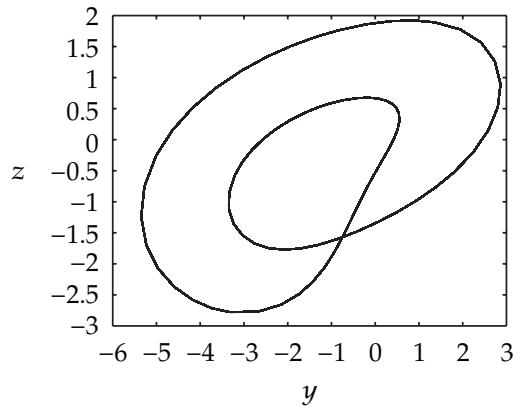

(b)

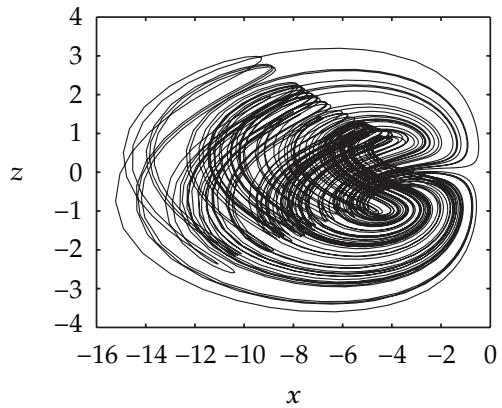

(d)

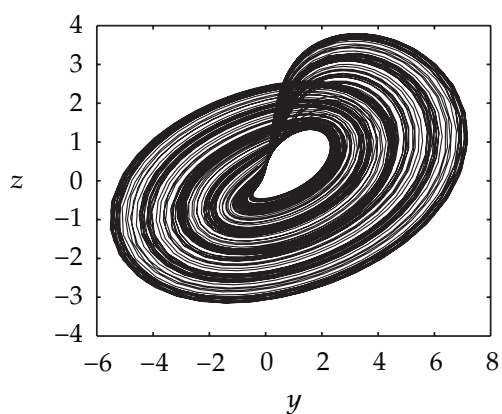

(f)

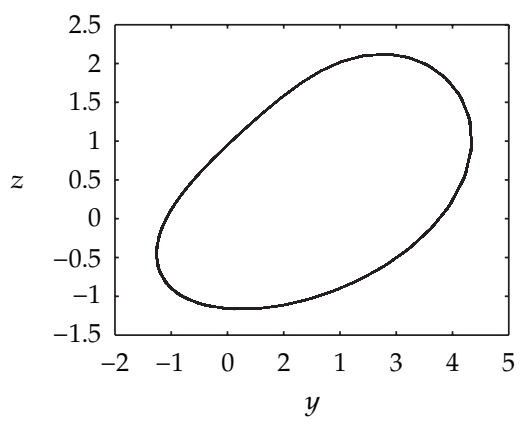

(h)

Figure 5: Phase portraits of the system (3.5) at (a) $u=-9$, (b) $u=-5.11$, (c) $u=-1.94$, (d) $u=-0.45$, (e) $u=0.45$, (f) $u=1.94$, (g) $u=5.11$, (h) $u=9$. 


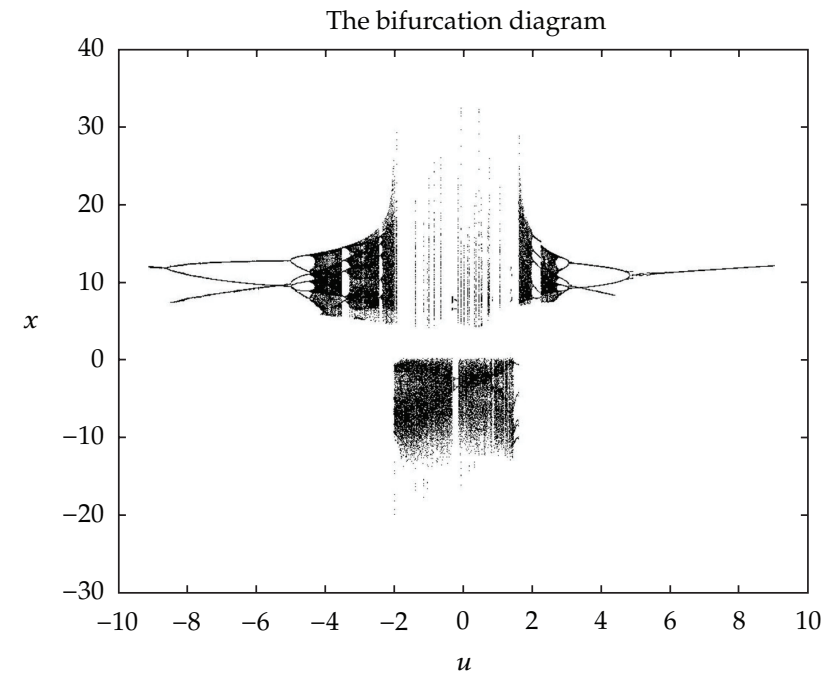

Figure 6: Bifurcation diagram of system states versus parameter $-9 \leq u \leq 9$.

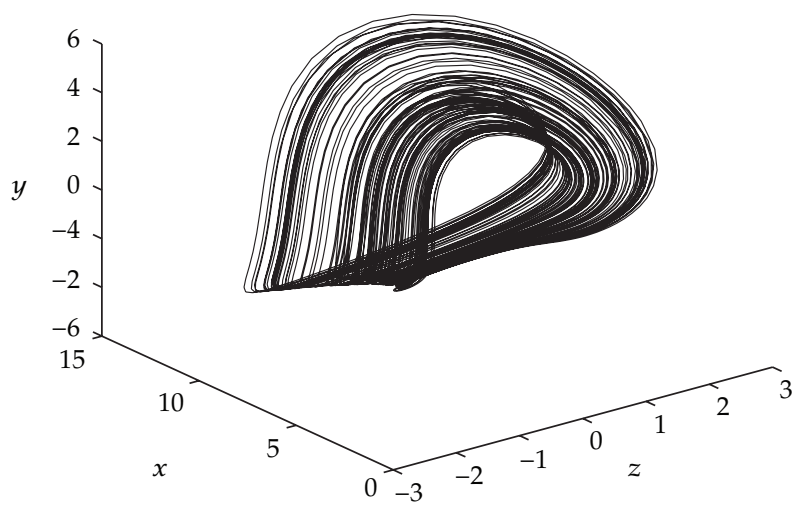

Figure 7: The new one-scroll chaotic attractor.

\subsection{The Left and Right Two-Scroll Chaotic Attractor}

With parameters $a=2.4, b=-3, c=14, d=-11, e=4, f=6, g=-1$, the system (2.3) can exhibit a right two-scroll chaotic attractor (see Figure 8), and with parameters $a=2.4, b=-3$, $c=14, d=-11, e=4, f=5.5, g=-1$, the system (2.3) can exhibit a left two-scroll chaotic attractor (see Figure 9).

\section{Poincaré Map, Bifurcation Diagram, and the Maximum Lyapunov Exponent Spectrum of the New Chaotic System}

Varying the parameter $f$, Poincare mapping of the chaotic attractors of the systems (2.3) are shown in Figures 10(a), 10(b), 10(c), and 10(d), respectively. Several sheets of the attractors are displayed. It is noticeable that the Poincare map of many chaotic systems such as the generalized Lorenz system [19] only shows a branch with several twigs. The Poincare map 


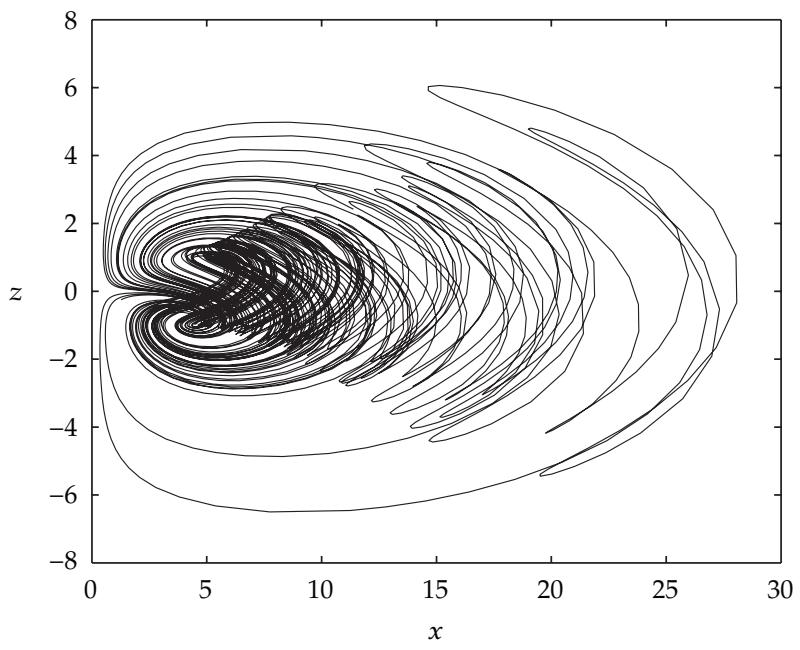

Figure 8: The new right two-scroll chaotic attractor.

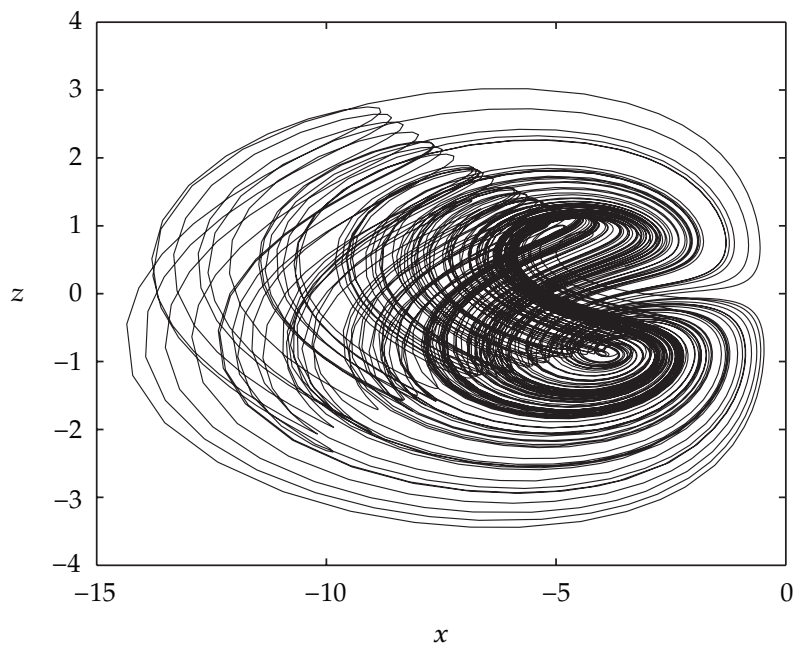

Figure 9: The new left two-scroll chaotic attractor.

in Figures 10(a), 10(b), 10(c) and 10(d), however, consists of virtually symmetrical branches and a number of nearly symmetrical twigs.

The bifurcation diagram would be far better to summarize all of the possible behaviors as the parameter varies on one diagram. For $1 \leq f \leq 9.4$ the bifurcation diagram of system (2.3) shows the complicated bifurcation phenomena (see Figure 11).

\section{Conclusions}

In this paper, a new four-scroll chaotic attractor in 3D autonomous system has been reported and confirmed analytically and numerically. In comparison with that of existing two-scroll or four-scroll chaotic attractors in 3D autonomous system, the novel chaotic attractor can generate four-scroll that consisted of the two-scroll transient chaotic and the two-scroll chaotic. The particular interest is that this novel system can generate different scroll chaotic 


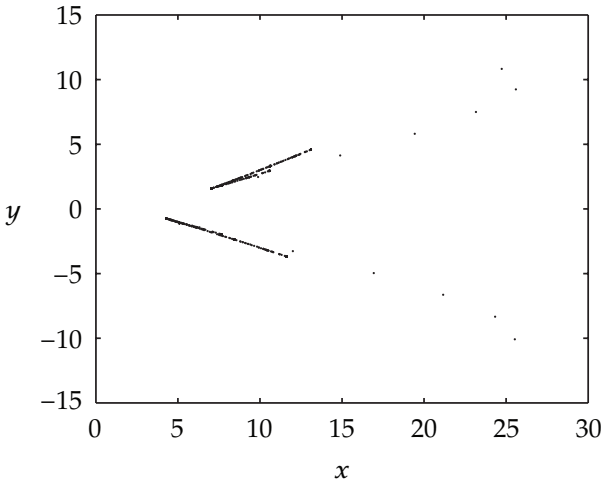

(a)

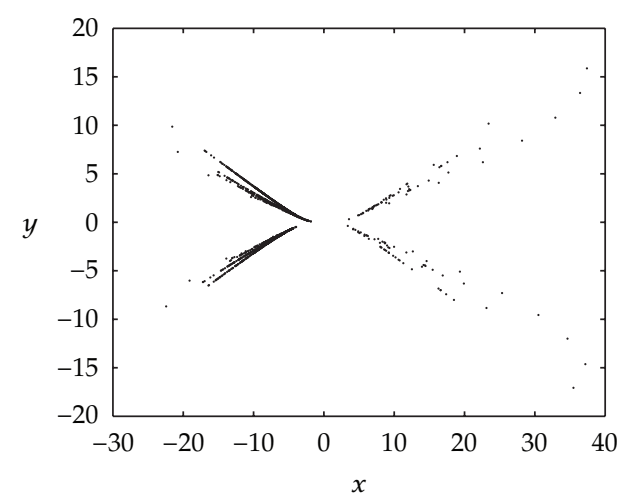

(c)

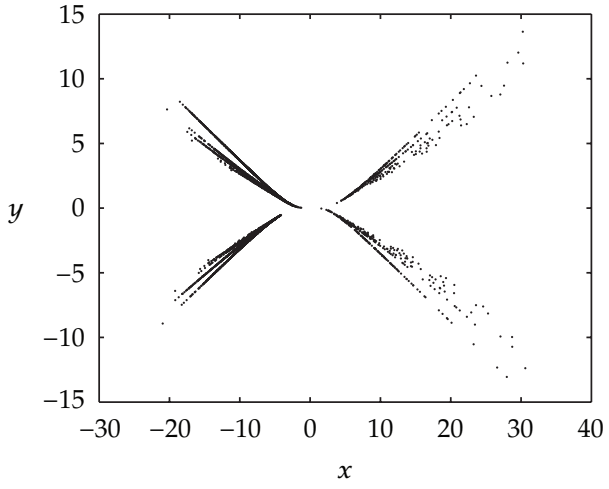

(b)

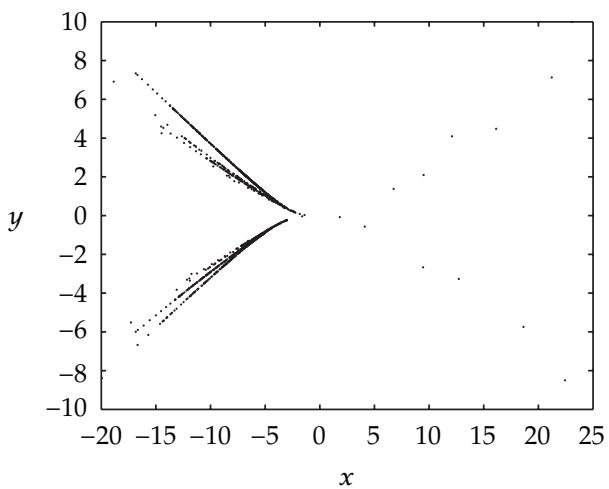

(d)

Figure 10: Poincaré maps of $x-y$ plane for $z=0$, (a) $f=7$, (b) $f=6$, (c) $f=5.58$, (d) $f=5.5$.

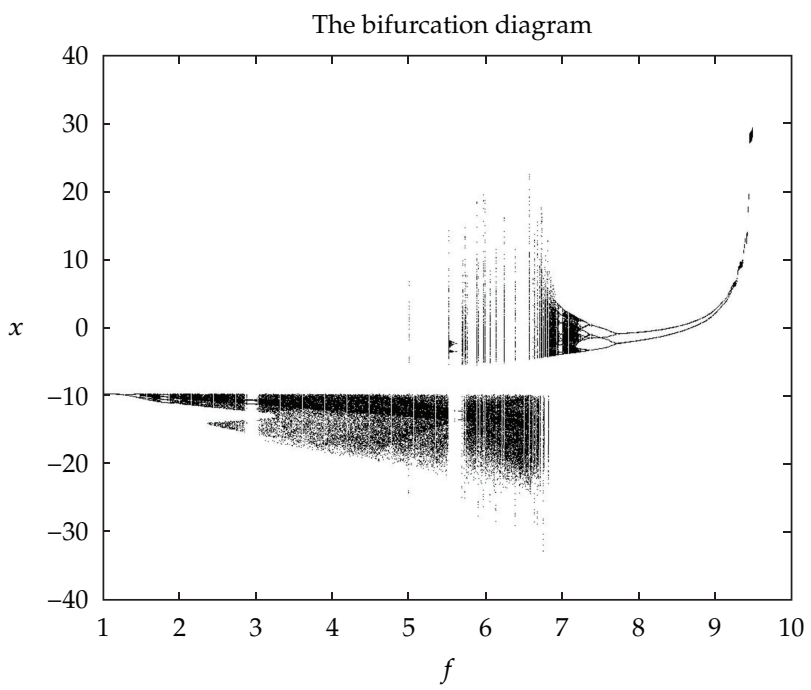

Figure 11: Bifurcation diagram of system states versus parameter $1 \leq f \leq 9.4$. 
attractors with variation of a single parameter. The topological structure of the new system should be completely and thoroughly investigated. It is expected that more detailed theory analyses and simulation investigations will be provided in a forthcoming paper.

\section{Acknowledgments}

This research is supported by the National Natural Science Foundation of China (61075060), the Innovation Program of Shanghai Municipal Education Commission (12zz064), the Key Basic Research Program of Shanghai City (09JC1400700), the Science and Technology Research Key Program for the Education Department of Hubei Province of China (D20105001, D20126004) and China Postdoctoral Science Foundation (2012M511259).

\section{References}

[1] E. N. Lorenz, "Deterministic non-periodic flows," Journal of the Atmospheric Sciences, vol. 20, pp. 130$148,1963$.

[2] O. E. Rössler, "An equation for continuous chaos," Physics Letters A, vol. 57, no. 4, pp. 397-398, 1976.

[3] G. Chen and T. Ueta, "Yet another chaotic attractor," International Journal of Bifurcation and Chaos in Applied Sciences and Engineering, vol. 9, no. 7, pp. 1465-1466, 1999.

[4] J. Lü and G. Chen, "A new chaotic attractor coined," International Journal of Bifurcation and Chaos in Applied Sciences and Engineering, vol. 12, no. 3, pp. 659-661, 2002.

[5] C. Liu, T. Liu, L. Liu, and K. Liu, "A new chaotic attractor," Chaos, Solitons and Fractals, vol. 22, no. 5, pp. 1031-1038, 2004.

[6] J. Lü, T. Zhou, G. Chen, and X. Yang, "Generating chaos with a switching piecewise-linear controller," Chaos, vol. 12, no. 2, pp. 344-349, 2002.

[7] W. Liu, W. K. S. Tang, and G. Chen, "2 2 2-scroll attractors generated in a three-dimensional smooth autonomous system," International Journal of Bifurcation and Chaos, vol. 17, no. 11, pp. 4153-4157, 2007.

[8] J. Lü and G. Chen, "Generating multiscroll chaotic attractors: theories, methods and applications," International Journal of Bifurcation and Chaos in Applied Sciences and Engineering, vol. 16, no. 4, pp. 775858, 2006.

[9] A.S. Elwakil, S. Özoğuz, and M. P. Kennedy, "A four-wing butterfly attractor from a fully autonomous system," International Journal of Bifurcation and Chaos in Applied Sciences and Engineering, vol. 13, no. 10, pp. 3093-3098, 2003.

[10] W. Liu and G. Chen, "A new chaotic system and its generation," International Journal of Bifurcation and Chaos in Applied Sciences and Engineering, vol. 13, no. 1, pp. 261-267, 2003.

[11] G. Qi, G. Chen, S. Li, and Y. Zhang, "Four-wing attractors: from pseudo to real," International Journal of Bifurcation and Chaos in Applied Sciences and Engineering, vol. 16, no. 4, pp. 859-885, 2006.

[12] J. Lü, G. Chen, and D. Cheng, "A new chaotic system and beyond: the generalized Lorenz-like system," International Journal of Bifurcation and Chaos in Applied Sciences and Engineering, vol. 14, no. 5, pp. 1507-1537, 2004.

[13] S. Dadras and H. R. Momeni, "A novel three-dimensional autonomous chaotic system generating two, three and four-scroll attractors," Physics Letters A, vol. 373, no. 40, pp. 3637-3642, 2009.

[14] A. Chen, J. Lu, J. Lü, and S. Yu, "Generating hyperchaotic Lü attractor via state feedback control," Physica A, vol. 364, pp. 103-110, 2006.

[15] G. Qi, G. Chen, S. Du, Z. Chen, and Z. Yuan, "Analysis of a new chaotic system," Physica A, vol. 352, no. 2-4, pp. 295-308, 2005.

[16] G. Qi, G. Chen, M. A. van Wyk, B. J. van Wyk, and Y. Zhang, "A four-wing chaotic attractor generated from a new 3-D quadratic autonomous system," Chaos, Solitons and Fractals, vol. 38, no. 3, pp. 705-721, 2008.

[17] J. C. Sprott, "Some simple chaotic flows," Physical Review E, vol. 50, no. 2, pp. R647-R650, 1994.

[18] T. Ueta and G. Chen, "Bifurcation analysis of Chen's equation," International Journal of Bifurcation and Chaos, vol. 10, pp. 917-931, 2009.

[19] S. Čelikovský and G. Chen, "On the generalized Lorenz canonical form," Chaos, Solitons and Fractals, vol. 26, no. 5, pp. 1271-1276, 2005.

[20] A. Wolf, J. B. Swift, H. L. Swinney, and J. A. Vastano, “Determining Lyapunov exponents from a time series," Physica D, vol. 16, no. 3, pp. 285-317, 1985. 


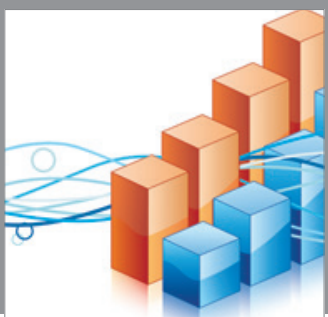

Advances in

Operations Research

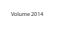

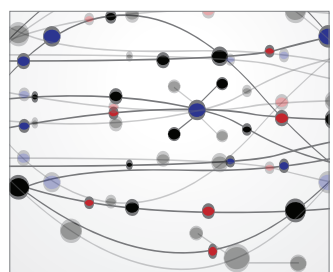

\section{The Scientific} World Journal
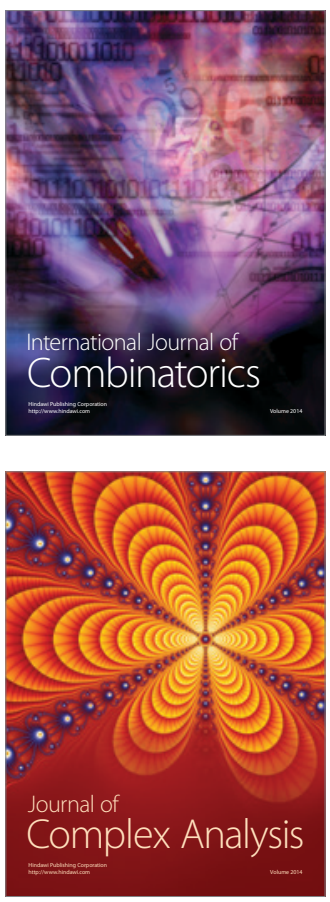

International Journal of

Mathematics and

Mathematical

Sciences
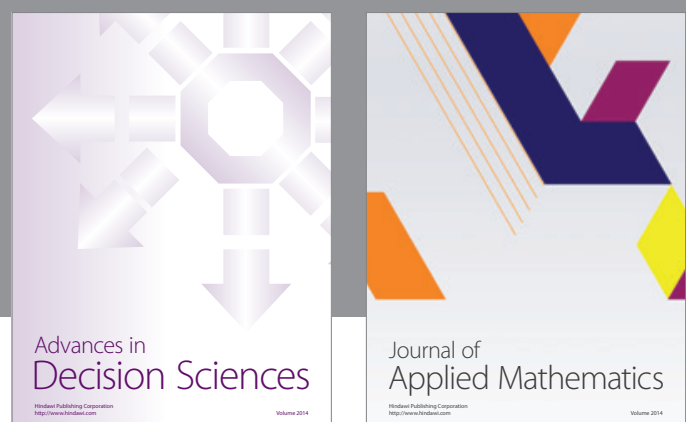

Journal of

Applied Mathematics
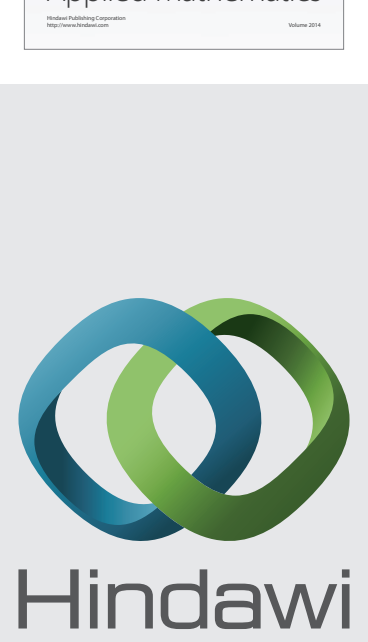

Submit your manuscripts at http://www.hindawi.com
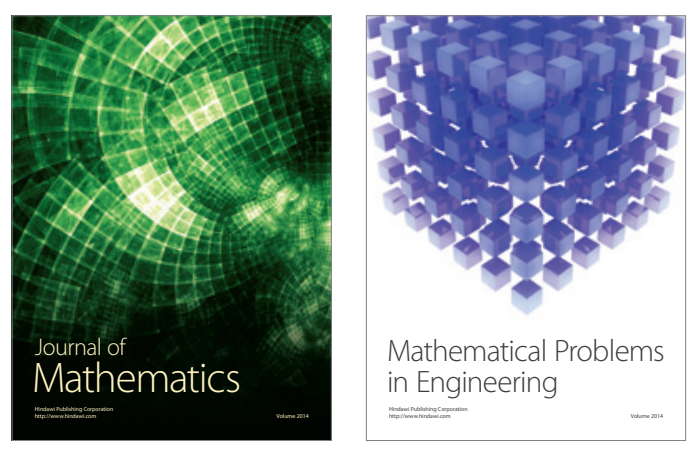

Mathematical Problems in Engineering
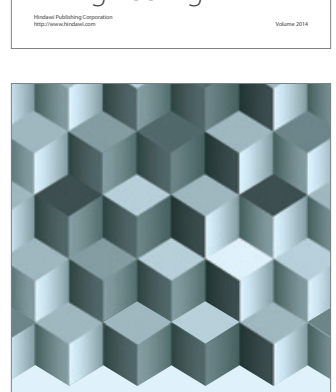

Journal of

Function Spaces
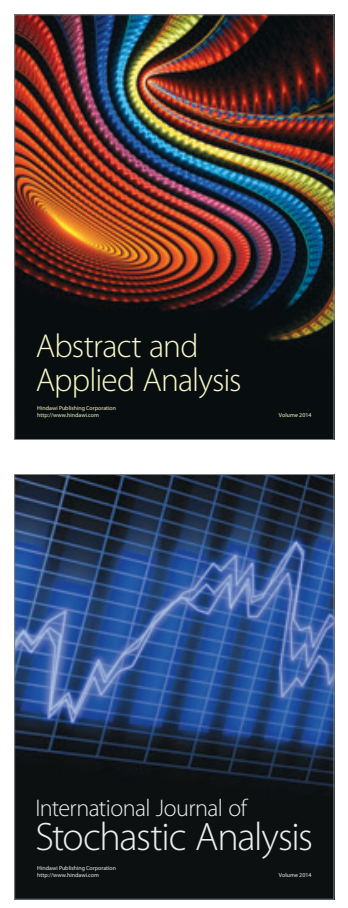

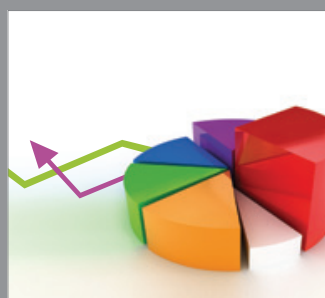

ournal of

Probability and Statistics

Promensencen
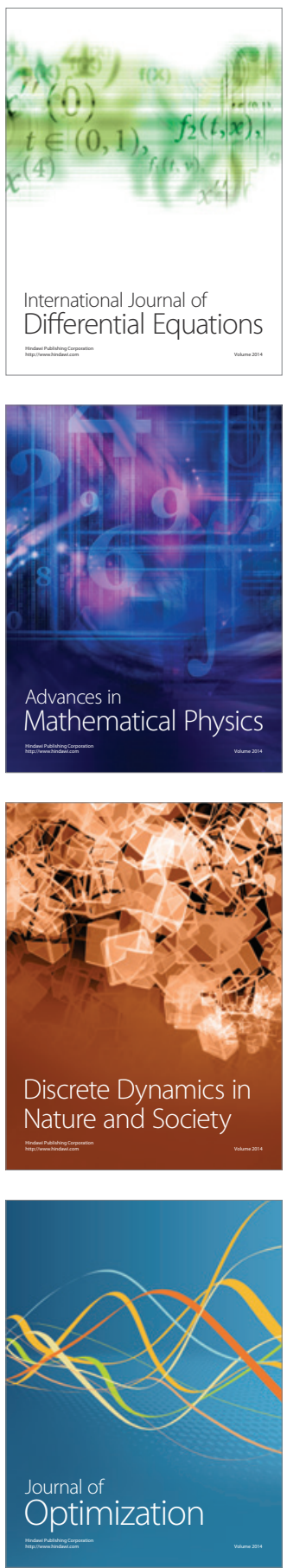